\title{
ON THE PERIODIC SOLUTIONS OF PERTURBED 4D NON-RESONANT SYSTEMS
}

\author{
JAUME LLIBRE ${ }^{1}$, DOUGLAS D. NOVAES ${ }^{2}$ AND MARCO ANTONIO TEIXEIRA ${ }^{2}$
}

\begin{abstract}
We provide sufficient conditions for the existence of periodic solutions of a 4D non-resonant system perturbed by smooth or non-smooth functions. We apply these results to study the small amplitude periodic solutions of the non-linear planar double pendulum perturbed by smooth or non-smooth function.
\end{abstract}

\section{IntRoduCtion}

A major problem in general perturbation theory is to detect how persistent are some given properties. In other words we want to translate some dynamical properties from the unperturbed system to the perturbed one. Often the unperturbed system is linear and the objects to be continued to the perturbed system are equilibrium points, periodic orbits or invariant torus. In this direction, the aim of this paper, is to deal with the periodic solutions of the following kind of differential systems

$$
\begin{aligned}
& x^{\prime}(t)=\omega_{1} y+\varepsilon P_{1}(t, x, y, z, w)+\varepsilon^{2} R_{1}(t, x, y, z, w, \varepsilon), \\
& y^{\prime}(t)=-\omega_{1} x+\varepsilon P_{2}(t, x, y, z, w)+\varepsilon^{2} R_{2}(t, x, y, z, w, \varepsilon), \\
& z^{\prime}(t)=\omega_{2} w+\varepsilon P_{3}(t, x, y, z, w)+\varepsilon^{2} R_{3}(t, x, y, z, w, \varepsilon), \\
& w^{\prime}(t)=-\omega_{2} z+\varepsilon P_{4}(t, x, y, z, w)+\varepsilon^{2} R_{4}(t, x, y, z, w, \varepsilon),
\end{aligned}
$$

where

$$
\begin{aligned}
& P_{i}(t, x, y, z, w)=P_{i}^{1}(t, x, y, z, w)+\operatorname{sign}\left(h_{i}(t, x, y, z, w)\right) P_{i}^{2}(t, x, y, z, w), \text { and } \\
& R_{i}(t, x, y, z, w, \varepsilon)=R_{i}^{1}(t, x, y, z, w, \varepsilon)+\operatorname{sign}\left(h_{i}(t, x, y, z, w)\right) R_{i}^{2}(t, x, y, z, w, \varepsilon) .
\end{aligned}
$$

Here $P_{i}^{j}: \mathbb{R} \times D \rightarrow \mathbb{R}, R_{i}^{j}: \mathbb{R} \times D \times\left(-\varepsilon_{0}, \varepsilon_{0}\right) \rightarrow \mathbb{R}$ for $i=1,2,3,4$ and $j=1,2$ are smooth functions $T$-periodic in the variable $t$ being $T$ the period of some periodic solution of (1) when $\varepsilon=0$, and $D$ an open subset of $\mathbb{R}^{4}$. The functions $h_{i}$ for $i=1,2,3,4$ are smooth having 0 as a regular value. The prime in (1) denotes derivative with respect to time $t$. We denote by $\Sigma=\left\{(t, x, y, z, w) \in \mathbb{R} \times D:\left(h_{1} h_{2} h_{3} h_{4}\right)(t, x, y, z)=0\right\}$ the set of discontinuity. Furthermore

Corresponding author Douglas D. Novaes: Departamento de Matematica, Universidade Estadual de Campinas, Caixa Postal 6065, 13083-859, Campinas, SP, Brazil. Tel. +55 19 91939832, Fax. +55 1935216094 , email: ddnovaes@gmail.com

2010 Mathematics Subject Classification. 37G15, 34C15, 37C30.

Key words and phrases. periodic solution, averaging theory, non-smooth dynamical systems, non-resonant systems, double pendulum. 
we assume that $h_{1}=h_{2}$ and $h_{3}=h_{4}$. The function $\operatorname{sgn}(z)$ denotes the sign function, i.e.

$$
\operatorname{sgn}(z)=\left\{\begin{array}{cc}
-1 & \text { if } z<0 \\
0 & \text { if } z=0 \\
1 & \text { if } z>0
\end{array}\right.
$$

Observe that system (1) with $\varepsilon=0$ is simply the unperturbed system. Otherwise we have the perturbed system.

Our goal is to provide an algorithm, via tools in advanced averaging theory, for detecting the periodic orbits of the perturbed system which emerge from the set of periodic orbits of the unperturbed system. It is to be noted that a very similar scenario holds for a model of the double pendulum in which our main results will be applied.

1.1. Background and historical facts. The first serious proofs in the averaging theory for differential systems can be traced back to the works of Fatou [6] in 1928, Krylov and Bogoliubov [4] in the 1930s and Bogoliubov [3] in 1945. For a modern point of view on this theory see the book of Sanders and Verhulst [13].

The method of averaging plays an important role in the study of nonlinear systems related to complex behavior patterns such as bifurcation and stability of the periodic solutions of such systems. Here we need to deal with systems in the normal form of the averaging theory given by

$$
\dot{\mathbf{x}}(t)=G_{0}(t, \mathbf{x})+\varepsilon G_{1}(t, \mathbf{x})+\varepsilon^{2} G_{2}(t, \mathbf{x}, \varepsilon),
$$

see the basic results on this theory in section 3. One of the basic problems for applying the averaging theory is to write the system under study in the normal form (23).

Another tool used in this paper is the regularization process for discontinuous differential systems introduced by Sotomayor and Teixeira in [14]. In this process a discontinuous vector field $Z(t, x)$ is approximated by an one-parameter family of continuous vector fields $Z_{\delta}(t, x)$.

As far as we know, the two methods cited above has been firstly used together in [10] by Llibre and Teixeixa where it was studied the stable limit cycle of a weight-driven pendulum clock. In this paper we use the ideas presented in [10] to study the periodic solutions of (1).

1.2. Setting the problem. The objective of this paper is to provide a system of equations whose simple zeros provide periodic solutions of (1). In order to present our results we need some preliminary definitions and notations.

The unperturbed system (1) has the origin as its unique singular point with eigenvalues $\pm \omega_{1} i, \pm \omega_{2} i$. If the non-resonant condition $\omega_{1} / \omega_{2} \in \mathbb{R} \backslash \mathbb{Q}$ is satisfied then this system has, in the phase space $(x, y, z, w)$, two planes filled with periodic solutions except the origin. The periods of such periodic orbits are

$$
T_{1}=\frac{2 \pi}{\omega_{1}} \quad \text { or } \quad T_{2}=\frac{2 \pi}{\omega_{2}} .
$$

These periodic orbits live into the planes associated to the eigenvectors with eigenvalues $\pm \omega_{1} i$ or $\pm \omega_{2} i$, respectively. We shall study which of these periodic solutions persist for the perturbed system (1) when the parameter $\varepsilon$ is sufficiently small and the functions of perturbation are either $T_{1}$ or $T_{2}$ periodic.

Let $\varphi_{1}(t, u, v)$ be the periodic function

$$
\varphi_{1}(t, u, v)=\left(u \cos \left(\omega_{1} t\right)+v \sin \left(\omega_{1} t\right), v \cos \left(\omega_{1} t\right)-u \sin \left(\omega_{1} t\right), 0,0\right) .
$$


Define $\mathcal{F}_{1}(x, y)$ :

$$
\begin{aligned}
& \int_{0}^{T_{1}}\left(\cos \left(\omega_{1} t\right) P_{1}^{1}\left(t, \varphi_{1}(t, x, y)\right)-\sin \left(\omega_{1} t\right) P_{2}^{1}\left(t, \varphi_{1}(t, x, y)\right)\right) d t \\
& +\int_{0}^{T_{1}}\left(\cos \left(\omega_{1} t\right) P_{1}^{2}\left(t, \varphi_{1}(t, x, y)\right)-\sin \left(\omega_{1} t\right) P_{2}^{2}\left(t, \varphi_{1}(t, x, y)\right)\right) \operatorname{sign}\left(h_{1}\left(t, \varphi_{1}(t, x, y)\right)\right) d t
\end{aligned}
$$

and $\mathcal{F}_{2}(x, y)$ :

$$
\begin{aligned}
& \int_{0}^{T_{1}}\left(\cos \left(\omega_{1} t\right) P_{2}^{1}\left(t, \varphi_{1}(t, x, y)\right)+\sin \left(\omega_{1} t\right) P_{1}^{1}\left(t, \varphi_{1}(t, x, y)\right)\right) d t \\
& +\int_{0}^{T_{1}}\left(\cos \left(\omega_{1} t\right) P_{2}^{2}\left(t, \varphi_{1}(t, x, y)\right)-\sin \left(\omega_{1} t\right) P_{1}^{2}\left(t, \varphi_{1}(t, x, y)\right)\right) \operatorname{sign}\left(h_{1}\left(t, \varphi_{1}(t, x, y)\right)\right) d t
\end{aligned}
$$

Now let $\varphi_{2}(t, u, v)$ be the periodic function

$$
\varphi_{2}(t, u, v)=\left(0,0, u \cos \left(\omega_{2} t\right)+v \sin \left(\omega_{2} t\right), v \cos \left(\omega_{2} t\right)-u \sin \left(\omega_{2} t\right)\right) .
$$

Define $\mathcal{F}^{1}(z, w)$ :

$$
\begin{aligned}
& \int_{0}^{T_{2}}\left(\cos \left(\omega_{2} t\right) P_{3}^{1}\left(t, \varphi_{2}(t, x, y)\right)-\sin \left(\omega_{2} t\right) P_{4}^{1}\left(t, \varphi_{1}(t, x, y)\right)\right) d t \\
& +\int_{0}^{T_{2}}\left(\cos \left(\omega_{2} t\right) P_{3}^{2}\left(t, \varphi_{2}(t, x, y)\right)-\sin \left(\omega_{2} t\right) P_{4}^{2}\left(t, \varphi_{1}(t, x, y)\right)\right) \operatorname{sign}\left(h_{3}\left(t, \varphi_{2}(t, x, y)\right)\right) d t
\end{aligned}
$$

and $\mathcal{F}^{2}(z, w)$ :

$$
\begin{aligned}
& \int_{0}^{T_{2}}\left(\cos \left(\omega_{2} t\right) P_{4}^{1}\left(t, \varphi_{2}(t, x, y)\right)+\sin \left(\omega_{2} t\right) P_{3}^{1}\left(t, \varphi_{2}(t, x, y)\right)\right) d t \\
& +\int_{0}^{T_{2}}\left(\cos \left(\omega_{2} t\right) P_{4}^{2}\left(t, \varphi_{2}(t, x, y)\right)-\sin \left(\omega_{2} t\right) P_{3}^{2}\left(t, \varphi_{2}(t, x, y)\right)\right) \operatorname{sign}\left(h_{3}\left(t, \varphi_{2}(t, x, y)\right)\right) d t
\end{aligned}
$$

A zero $\left(x^{*}, y^{*}\right)$ of the system of functions

$$
\mathcal{F}_{1}(x, y)=0, \quad \mathcal{F}_{2}(x, y)=0,
$$

such that

$$
\operatorname{det}\left(\left.\frac{\partial\left(\mathcal{F}_{1}, \mathcal{F}_{2}\right)}{\partial(x, y)}\right|_{(x, y)=\left(x^{*}, y^{*}\right)}\right) \neq 0,
$$

is called a simple zero of (9). Similarly, we define a simple zero of the system of functions

$$
\mathcal{F}^{1}(z, w)=0, \quad \mathcal{F}^{2}(z, w)=0 .
$$

Remark 1. For $p \in D$, let $\varphi(t, p)$ be the solution of $(1)$ such that $\varphi(0, p)=p$. We say that the Crossing Hypothesis is satisfied if there exists a compact set $V \subset D$ such that the curve $t \mapsto(t, \varphi(t, p))$ reaches the set $\Sigma$ at points of crossing regions (see Appendix) for every $p \in V$ and $t \in[0, T]$.

1.3. Statement of results. Our main result on the periodic solutions of the perturbed system (1) which bifurcate from the periodic solutions of the unperturbed system with period $T_{1}$ is the following.

Theorem A. Assume that the functions $P_{i}^{j}$ and $R_{i}^{j}$ of (1) are $T_{1}$-periodic in the variable $t$. Also assume that the Crossing Hypothesis (see Remark 1) is satisfied for $\varepsilon \in\left(0, \varepsilon_{0}\right)$ with $\varepsilon_{0}>0$ and $(x, y, z, w) \in V$. Then for $\varepsilon>0$ sufficiently small and for every simple zero $\left(x^{*}, y^{*}\right)$ of $(9)$ such that $\left(x^{*}, y^{*}, 0,0\right) \in V$, the perturbed system (1) has a $T_{1}$-periodic solution $\varphi(t, \varepsilon)$ such that $\varphi(0, \varepsilon) \rightarrow\left(x^{*}, y^{*}, 0,0\right)$ when $\varepsilon \rightarrow 0$. 
For $\varepsilon>0$ sufficiently small the solution $\varphi(t, \varepsilon)$ of Theorem A is close to the plane defined by the eigenvectors of the eigenvalues $\pm i \omega_{1}$.

With a change of variables we can also state a result on the periodic solutions of perturbed system (1) which bifurcate from the periodic solutions of the unperturbed system with period $T_{2}$.

Corollary 1. Assume that the functions $P_{i}^{j}$ and $R_{i}^{j}$ of (1) are $T_{2}$-periodic in the variable $t$. Also assume that the Crossing Hypothesis (see Remark 1) is satisfied for $\varepsilon \in\left(0, \varepsilon_{0}\right)$ with $\varepsilon_{0}>0$. and $(x, y, z, w) \in V$. Then for $\varepsilon>0$ sufficiently small and for every simple zero $\left(z^{*}, w^{*}\right)$ of (10) such that $\left(0,0, z^{*}, w^{*}\right) \in V$, the perturbed system (1) has a $T_{2}$-periodic solution $\varphi(t, \varepsilon)$ such that $\varphi(0, \varepsilon) \rightarrow\left(0,0, z^{*}, w^{*}\right)$ when $\varepsilon \rightarrow 0$.

Again, for $\varepsilon>0$ sufficiently small the solution $\varphi(t, \varepsilon)$ of Corollary 1 is close to the plane defined by the eigenvectors of the eigenvalues $\pm i \omega_{2}$.

Theorem A and Corollary 1 are proved in Section 4. Its proof is based in the averaging theory for computing periodic solutions, see Section 3.

Remark 2. The theory could be developed for a more general system by considering the smooth functions $P_{i}^{j}$ and $R_{i}^{j}, T_{P_{i}^{j}}$-periodic and $T_{R_{i}^{j}}$-periodic in the variable $t$ and respectively in resonance $p_{P_{i}^{j}}: q_{P_{i}^{j}}$ and $p_{R_{i}^{j}}: q_{R_{i}^{j}}$ with some of the periodic solutions of the unperturbed system (1), being $p$ and $q$ relatively prime positive integers for $p=p_{P_{i}^{j}}, p_{R_{i}^{j}}, q=q_{P_{i}^{j}}, q_{R_{i}^{j}}, i=1,2,3,4$ and $j=1,2$. However, in this case we may assume that the functions $P_{i}^{j}$ and $R_{i}^{j}$ for $i=1,2,3,4$ and $j=1,2$ are $k T$-periodic being $T$ the period of the periodic solution of the unperturbed system (1) for which the functions are resonant. Indeed, if we take $k$ the least common multiple among $p_{P_{i}^{j}}$ and $p_{R_{i}^{j}}$ for $i=1,2,3,4$ and $j=1,2$, then there exists integers $n_{P_{i}^{j}}$ and $n_{R_{i}^{j}}$ such that $k=n_{P_{i}^{j}} p_{P_{i}^{j}}=n_{R_{i}^{j}} p_{R_{i}^{j}}$. Hence

$$
k T=n_{P_{i}^{j}} q_{P_{i}^{j}} \frac{p_{P_{i}^{j}}}{q_{P_{i}^{j}}} T=n_{R_{i}^{j}} q_{R_{i}^{j}} \frac{p_{R_{i}^{j}}}{q_{R_{i}^{j}}} T,
$$

for $i=1,2,3,4$, and $j=1,2$. Here to make the notation simpler we assume that all functions have the same period.

\section{Application: Double pendulum model}

The planar double pendulum model consists in a system of two point masses $m_{1}$ and $m_{2}$ moving in a fixed plane, in which the distance between a point $P$ (called pivot) and $m_{1}$ and the distance between $m_{1}$ and $m_{2}$ are fixed, and equal to $l_{1}$ and $l_{2}$ respectively. We assume the masses do not interact. We allow gravity to act on the masses $m_{1}$ and $m_{2}$.

The position of the double pendulum is determined by the two angles $\phi_{1}$ and $\phi_{2}$ shown in Figure 1. The corresponding Lagrange equations of motion are

$$
\begin{aligned}
& \left(m_{1}+m_{2}\right) l_{1} \ddot{\phi}_{1}+m_{2} l_{2} \ddot{\phi}_{2} \cos \left(\phi_{1}-\phi_{2}\right)+\left(m_{1}+m_{2}\right) g \sin \left(\phi_{1}\right) \\
& +m_{2} l_{2} \dot{\phi}_{2}^{2} \sin \left(\phi_{1}-\phi_{2}\right)=0, \\
& m_{2} l_{1} \ddot{\phi}_{1} \cos \left(\phi_{1}-\phi_{2}\right)+m_{2} l_{2} \ddot{\phi}_{2}+m_{2} g \sin (\phi 2)+m_{2} l_{1} \dot{\phi}_{1}^{2} \sin \left(\phi_{1}-\phi_{2}\right)=0,
\end{aligned}
$$

where $g$ is the acceleration of the gravity. For more details on these equations of motion see [8]. Here the dot denotes derivative with respect to the time $\tau$.

The authors in [9] have studied, in the vicinity of the equilibrium $\phi_{1}=\phi_{2}=0$, the persistence of periodic solutions of system (11) perturbed smoothly in the particular case when $m_{1}=m_{2}$ and $l_{1}=l_{2}$. In this paper stronger generalizations are considered. 


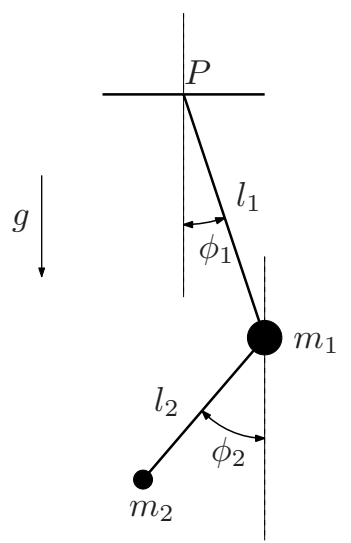

Figure 1. The planar double pendulum.

Denote the expressions for $\ddot{\phi}_{1}$ and $\ddot{\phi}_{2}$ in (11) respectively by $H_{1}\left(\phi_{1}, \dot{\phi}_{1}, \phi_{2}, \dot{\phi}_{2}\right)$ and $H_{2}\left(\phi_{1}, \dot{\phi}_{1}\right.$, $\left.\phi_{2}, \dot{\phi}_{2}\right)$. In this section we consider the following perturbed problem:

$$
\begin{aligned}
\ddot{\phi}_{1}(\tau)= & H_{1}\left(\phi_{1}, \dot{\phi}_{1}, \phi_{2}, \dot{\phi}_{2}\right)+\varepsilon\left(F_{1}\left(\tau, \phi_{1}, \dot{\phi}_{1}, \phi_{2}, \dot{\phi}_{2}\right)+\operatorname{sgn}\left(\phi_{1}\right) F_{2}\left(\tau, \phi_{1}, \dot{\phi}_{1}, \phi_{2}, \dot{\phi}_{2}\right)\right) \\
& +\varepsilon^{2}\left(G_{1}\left(\tau, \phi_{1}, \dot{\phi}_{1}, \phi_{2}, \dot{\phi}_{2}\right)+\operatorname{sgn}\left(\phi_{1}\right) G_{2}\left(\tau, \phi_{1}, \dot{\phi}_{1}, \phi_{2}, \dot{\phi}_{2}\right)\right)+\mathcal{O}\left(\varepsilon^{3}\right), \\
\ddot{\phi}_{2}(\tau)= & H_{2}\left(\phi_{1}, \dot{\phi}_{1}, \phi_{2}, \dot{\phi}_{2}\right)+\varepsilon\left(F_{3}\left(\tau, \phi_{1}, \dot{\phi}_{1}, \phi_{2}, \dot{\phi}_{2}\right)+\operatorname{sgn}\left(\phi_{2}\right) F_{4}\left(\tau, \phi_{1}, \dot{\phi}_{1}, \phi_{2}, \dot{\phi}_{2}\right)\right) \\
& +\varepsilon^{2}\left(G_{3}\left(\tau, \phi_{1}, \dot{\phi}_{1}, \phi_{2}, \dot{\phi}_{2}\right)+\operatorname{sgn}\left(\phi_{2}\right) G_{4}\left(\tau, \phi_{1}, \dot{\phi}_{1}, \phi_{2}, \dot{\phi}_{2}\right)\right)+\mathcal{O}\left(\varepsilon^{3}\right) .
\end{aligned}
$$

The smooth functions $F_{i}$ and $G_{i}$ for $i=1,2,3,4$ define the perturbation. These functions are respectively $T$-periodic in the variable $\tau$, being $T$ the period of some periodic solution of (12) when $\varepsilon=0$, if there exists. We also assume that $F_{i}(\tau, 0,0,0,0)=0$ for $i=1,2,3,4$.

Roughly speaking, the functions $F_{i}$ and $G_{i}$ for $i=1,2,3,4$, can be taken in a certain way arbitrary. It makes us able to provide, in a physical context, the real meaning of these functions. In our case, since we are working with discontinuities in the variables $\phi_{1}$ and $\phi_{2}$, the functions $F_{1}, F_{2}, G_{1}$ and $G_{2}$ could model the escapement for the particle $m_{1}$, and the functions $F_{3}, F_{4}$, $G_{3}$ and $G_{4}$ could model the escapement for the particle $m_{2}$. If discontinuities in the variables $\dot{\phi}_{1}$ and $\dot{\phi}_{2}$ are considered instead discontinuities in the variables $\phi_{1}$ and $\phi_{2}$, the respective functions could model the Coulomb Friction. We can also work by composing these two phenomena. For more details on physical systems with discontinuous models see, for instance, [1] and [2].

The objective of this section is to provide a system of equations whose simple zeros provide periodic solutions (see Figure 2) of the perturbed planar double pendulum (12). In order to present our results we need some preliminary definitions and notations.

In what follows we define the real parameters

$$
\gamma=\sqrt{\frac{l_{1} m_{1}}{g m_{2}}}, \quad a=\frac{m_{1}+m_{2}}{m_{2}}>1, \quad \text { and } \quad b=\frac{l_{1}\left(m_{1}+m_{2}\right)}{l_{2} m_{2}}>0,
$$

the frequencies

$$
\omega_{1}=\frac{\sqrt{a+b-\sqrt{\Delta}}}{\sqrt{2}} \text { and } \quad \omega_{2}=\frac{\sqrt{a+b+\sqrt{\Delta}}}{\sqrt{2}}
$$




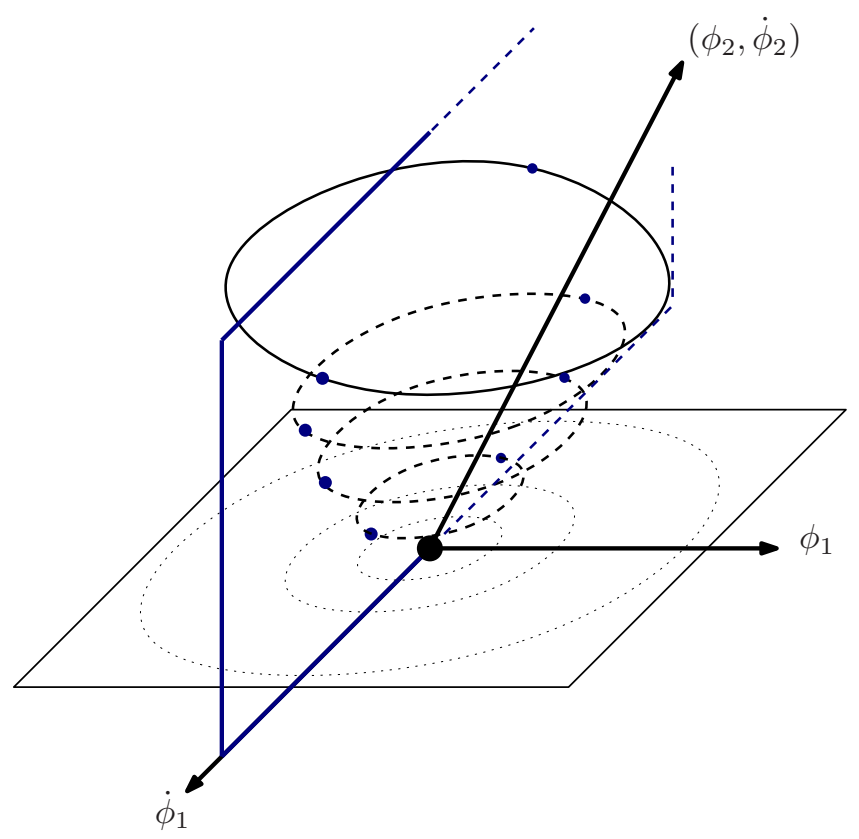

FiguRE 2. Periodic solution of the perturbed system (12) converging to the origin when $\varepsilon \rightarrow 0$.

with $\Delta=(a-b)^{2}+4 b>0$, and the periods

$$
T_{1}=\frac{2 \pi}{\omega_{1}} \quad \text { and } \quad T_{2}=\frac{2 \pi}{\omega_{2}}
$$

We shall study the persistence of periodic solutions for the perturbed system (12) when the parameter $\varepsilon$ is sufficiently small and the functions $F_{i}$ and $G_{i}$ for $i=1,2,3,4$ have period either $\gamma T_{1}$, or $\gamma T_{2}$.

Now let

$$
\tilde{F}_{i}\left(t, u_{1}, u_{2}, u_{3}, u_{4}\right)=d_{i}^{1}(t) u_{1}+d_{i}^{2}(t) u_{2}+d_{i}^{3}(t) u_{3}+d_{i}^{4}(t) u_{4},
$$

with

$$
\begin{array}{lll}
d_{i}^{1}(t) & =\gamma^{2} \frac{\partial F_{i}}{\partial \phi_{1}}(\gamma t, 0,0,0,0), & d_{i}^{2}(t)=\gamma \frac{\partial F_{i}}{\partial \dot{\phi}_{1}}(\gamma t, 0,0,0,0), \\
d_{i}^{3}(t)=\gamma^{2} \frac{\partial F_{i}}{\partial \phi_{2}}(\gamma t, 0,0,0,0), & d_{i}^{4}(t)=\gamma \frac{\partial F_{i}}{\partial \dot{\phi}_{2}}(\gamma t, 0,0,0,0),
\end{array}
$$

and let

$$
\tilde{G}_{i}(t)=\gamma^{2} G_{i}(\gamma t, 0,0,0,0)
$$


We define the functions:

$$
\begin{aligned}
\mathcal{F}_{1}(x, y)=\quad \int_{0}^{T_{1}} & \sin \left(\omega_{1} t\right)\left(2 b\left(\underline{F}_{1}+\tilde{G}_{1}(t)\right)+\left(\underline{F}_{3}+\tilde{G}_{3}(t)\right)(a-b+\sqrt{\Delta})\right) d t \\
+\int_{0}^{T_{1}} & \sin \left(\omega_{1} t\right)\left(2 b\left(\underline{F}_{2}+\tilde{G}_{2}(t)\right)\right. \\
& \left.+\left(\underline{F}_{4}+\tilde{G}_{4}(t)\right)(a-b+\sqrt{\Delta})\right) \operatorname{sgn}\left(\pi_{1} \circ \varphi_{1}(t, x, y)\right) d t,
\end{aligned}
$$

$$
\begin{aligned}
\mathcal{F}_{2}(x, y)=\quad \int_{0}^{T_{1}} & \cos \left(\omega_{1} t\right)\left(2 b\left(\underline{F}_{1}+\tilde{G}_{1}(t)\right)+\left(\underline{F}_{3}+\tilde{G}_{3}(t)\right)(a-b+\sqrt{\Delta})\right) d t \\
+\int_{0}^{T_{1}} & \cos \left(\omega_{1} t\right)\left(2 b\left(\underline{F}_{2}+\tilde{G}_{2}(t)\right)\right. \\
& \left.+\left(\underline{F}_{4}+\tilde{G}_{4}(t)\right)(a-b+\sqrt{\Delta})\right) \operatorname{sgn}\left(\pi_{1} \circ \varphi_{1}(t, x, y)\right) d t
\end{aligned}
$$

where

$$
\underline{F}_{i}=\tilde{F}_{i}\left(t, A_{1}, B_{1}, C_{1}, D_{1}\right)
$$

for $i=1,2,3,4$ with

$$
\begin{aligned}
& A_{1}=\frac{(-a+b+\sqrt{\Delta})}{2 b \omega_{1}}\left(x \cos \left(\omega_{1} t\right)+y \sin \left(\omega_{1} t\right)\right), \\
& B_{1}=\frac{(-a+b+\sqrt{\Delta})}{2 b}\left(y \cos \left(\omega_{1} t\right)-x \sin \left(\omega_{1} t\right)\right), \\
& C_{1}=\frac{1}{\omega_{1}}\left(x \cos \left(\omega_{1} t\right)+y \sin \left(\omega_{1} t\right)\right), \\
& D_{1}=y \cos \left(\omega_{1} t\right)-x \sin \left(\omega_{1} t\right),
\end{aligned}
$$

and $\pi_{1}$ is the projection onto the first coordinate.

We also define the functions:

$$
\begin{aligned}
\mathcal{F}^{1}(z, w)=\quad & \int_{0}^{T_{2}} \sin \left(\omega_{2} t\right)\left(-2 b\left(\bar{F}_{1}+\tilde{G}_{1}(t)\right)+\left(\bar{F}_{3}+\tilde{G}_{3}(t)\right)(-a+b+\sqrt{\Delta})\right) d t \\
+ & \int_{0}^{T_{2}} \sin \left(\omega_{2} t\right)\left(2 b\left(\bar{F}_{2}+\tilde{G}_{2}(t)\right)\right. \\
& \left.+\left(\bar{F}_{4}+\tilde{G}_{4}(t)\right)(-a+b+\sqrt{\Delta})\right) \operatorname{sgn}\left(\pi_{3} \circ \varphi_{2}(t, z, w)\right) d t, \\
\mathcal{F}^{2}(z, w)=\quad & \int_{0}^{T_{2}} \cos \left(\omega_{2} t\right)\left(-2 b\left(\bar{F}_{1}+\tilde{G}_{1}(t)\right)+\left(\bar{F}_{3}+\tilde{G}_{3}(t)\right)(a-b+\sqrt{\Delta})\right) d t \\
+ & \int_{0}^{T_{2}} \cos \left(\omega_{2} t\right)\left(2 b\left(\bar{F}_{2}+\tilde{G}_{2}(t)\right)\right. \\
& \left.+\left(\bar{F}_{4}+\tilde{G}_{4}(t)\right)(a-b+\sqrt{\Delta})\right) \operatorname{sgn}\left(\pi_{3} \circ \varphi_{2}(t, z, w)\right) d t .
\end{aligned}
$$

where

$$
\bar{F}_{i}=\tilde{F}_{i}\left(t, A_{2}, B_{2}, C_{2}, D_{2}\right)
$$


for $i=1,2,3,4$ with

$$
\begin{aligned}
& A_{2}=-\frac{(a-b+\sqrt{\Delta})}{2 b \omega_{2}}\left(z \cos \left(\omega_{2} t\right)+w \sin \left(\omega_{2} t\right)\right), \\
& B_{2}=-\frac{(a-b+\sqrt{\Delta})}{2 b}\left(w \cos \left(\omega_{2} t\right)-z \sin \left(\omega_{2} t\right)\right), \\
& C_{2}=\frac{1}{\omega_{2}}\left(z \cos \left(\omega_{2} t\right)+w \sin \left(\omega_{2} t\right)\right), \\
& D_{2}=w \cos \left(\omega_{2} t\right)-z \sin \left(\omega_{2} t\right),
\end{aligned}
$$

and $\pi_{3}$ is the projection onto the third coordinate.

Consider the systems

$$
\mathcal{F}_{1}(x, y)=0, \quad \mathcal{F}_{2}(x, y)=0,
$$

and

$$
\mathcal{F}^{1}(z, w)=0, \quad \mathcal{F}^{2}(z, w)=0 .
$$

In the next proposition we state a result on the existence of periodic solutions of the nonsmooth perturbed double pendulum (12).

Proposition 2. Assume that $F_{i}$ and $G_{i}$ of $(12)$ are $\gamma T_{1}$-periodic in the variable $t$. Also assume that the crossing hypothesis is satisfied for $\varepsilon \in\left(0, \varepsilon_{0}\right)$ with $\varepsilon_{0}>0$ and $(x, y, z, w) \in V$. Then for $\varepsilon>0$ sufficiently small and for every simple zero $\left(x^{*}, y^{*}, 0,0\right) \in V$ of the non-smooth system (21) such that the orbits pass by $D$, the non-smooth perturbed double pendulum (12) has a $\gamma T_{1}$-periodic solution $\varphi(t, \varepsilon)$ such that $\varphi(0, \varepsilon) \rightarrow(0,0,0,0)$ when $\varepsilon \rightarrow 0$.

In a similar way we can also state a result on the periodic solutions of the non-smooth perturbed double pendulum (12) which bifurcate from the periodic solutions of the unperturbed one with period $T_{2}$

Proposition 3. Assume that $F_{i}$ and $G_{i}$ of (12) are $\gamma T_{2}$-periodic in the variable $t$. Also assume that the crossing hypothesis is satisfied for $\varepsilon \in\left(0, \varepsilon_{0}\right)$ with $\varepsilon_{0}>0$ and $(x, y, z, w) \in V$. Then for $\varepsilon>0$ sufficiently small and for every simple zero $\left(0,0, z^{*}, w^{*}\right) \in V$ of the non-smooth system (22) such that the orbits pass by D, the non-smooth perturbed double pendulum (12) has a $\gamma T_{2}$-periodic solution $\varphi(t, \varepsilon)$ such that $\varphi(0, \varepsilon) \rightarrow(0,0,0,0)$ when $\varepsilon \rightarrow 0$.

We provide now an application of Propositions 2 and 3.

Corollary 4. Suppose that $F_{1}=\left(k_{1} / \gamma^{2}\right) z+f_{1}, F_{3}=\left(k_{3} / \gamma^{2}\right) x+f_{3}, G_{2}=1 / \gamma^{2}+g_{2}$, and $G_{4}=1 / \gamma^{2}+g_{4}$, where

$$
k_{1}=\frac{-\omega_{1}^{2}}{2 b \pi}, \quad \text { and } \quad k_{3}=\frac{-4 b \omega_{1}^{2}}{\pi\left((a-b)^{2}-\Delta\right)},
$$

with $f_{1}, f_{3}, g_{2}, g_{4}, F_{2}, F_{4}, G_{1}$, and $G_{3}$ being $\gamma T_{i}$-periodic functions in the variable $\tau$ having no linear terms and no constant terms in relation with the spatial variables. Then the differential system (12) for $|\varepsilon|>0$ sufficiently small has two $\gamma T_{i}$-periodic solution bifurcating from the origin. Here $i=1,2$.

Propositions 2 and 3, and Corollary 4 are proved in section 5 . 


\section{BASIC RESUlts ON AVERAGING THEORY}

For proving the main results of this paper we present the basic result from the averaging theory that we need here.

Consider the differential systems of the form

$$
\dot{\mathbf{x}}(t)=G_{0}(t, \mathbf{x})+\varepsilon G_{1}(t, \mathbf{x})+\varepsilon^{2} G_{2}(t, \mathbf{x}, \varepsilon),
$$

with $|\varepsilon|>0$ sufficiently small, where the functions $G_{0}, G_{1}: \mathbb{R} \times \Lambda \rightarrow \mathbb{R}^{n}$ and $G_{2}: \mathbb{R} \times \Lambda \times$ $\left(-\varepsilon_{0}, \varepsilon_{0}\right) \rightarrow \mathbb{R}^{n}$ are $\mathcal{C}^{2}$ functions, $T$-periodic in the variable $t$, and $\Lambda$ is an open subset of $\mathbb{R}^{n}$. Assume that the $T$-periodic solutions of the unperturbed system

$$
\dot{\mathbf{x}}(t)=G_{0}(t, \mathbf{x}),
$$

form a submanifold $\mathcal{Z}$ of dimension $k$ in $\mathbb{R}^{n}$. In the study of the periodic solutions the objective of the averaging theory is to detect which periodic solutions of the unperturbed system (24) persist as periodic solutions in the perturbed differential system (23) for $|\varepsilon|>0$ sufficiently small.

We denote by $\mathbf{x}(t, \mathbf{z}, \varepsilon)$ the solution of system $(24)$ such that $\mathbf{x}(0, \mathbf{z}, \varepsilon)=\mathbf{z}$. The first variational equation of system $(24)$ on the periodic solution $\mathbf{x}(t, \mathbf{z}, 0)$ is

$$
\dot{\mathbf{y}}=D_{\mathbf{x}} G_{0}(t, \mathbf{x}(t, \mathbf{z}, 0)) \mathbf{y}
$$

where $\mathbf{y}$ is a $n \times n$ matrix. From now on let $M_{\mathbf{z}}(t)$ be the fundamental matrix of system $(25)$ such tat $M_{\mathbf{z}}(0)=\mathrm{Id}$. Let $\xi: \mathbb{R}^{k} \times \mathbb{R}^{n-k} \rightarrow \mathbb{R}^{k}$ be the projection $\xi\left(x_{1}, \ldots, x_{n}\right)=\left(x_{1}, \ldots, x_{k}\right)$.

The $T$-periodic solutions of the perturbed system (24) coming from the unperturbed system (23) for $|\varepsilon|>0$ sufficiently small can be computed using the following result.

Theorem 5. We denote by $V$ an open and bounded subset of $\mathbb{R}^{k}$ containing the submanifold $\mathcal{Z}$, and we denote by $\beta: \mathrm{Cl}(V) \rightarrow \mathbb{R}^{n-k}$ a $\mathcal{C}^{2}$ function such that $\mathcal{Z}=\left\{\mathbf{z}_{\alpha}=(\alpha, \beta(\alpha)), \alpha \in \mathrm{Cl}(V)\right\} \subset$ $\Lambda$, where for every $\mathbf{z}_{\alpha} \in \mathcal{Z}$ the solution $\mathbf{x}\left(t, \mathbf{z}_{\alpha}\right)$ of (24) is T-periodic. Let $M_{\mathbf{z}_{\alpha}}(t)$ be fundamental matrix of (25) associated to the solution $\mathbf{x}\left(t, \mathbf{z}_{\alpha}\right)$. Assume that the matrix $M_{\mathbf{z}_{\alpha}}^{-1}(0)-M_{\mathbf{z}_{\alpha}}^{-1}(T)$ has in the lower right corner $a(n-k) \times(n-k)$ matrix $A_{\alpha}$ with $\operatorname{det}\left(A_{\alpha}\right) \neq 0$, and in the upper right corner the $k \times(n-k)$ zero matrix. Let $\mathcal{G}: \mathrm{Cl}(V) \rightarrow \mathbb{R}^{k}$ be the function defined by

$$
\mathcal{G}(\alpha)=\xi\left(\frac{1}{T} \int_{0}^{T} M_{\mathbf{z}_{\alpha}}^{-1}(t) G_{1}\left(t, \mathbf{x}\left(t, \mathbf{z}_{\alpha}\right)\right) d t\right) .
$$

If there is $a \in V$ such that $\mathcal{G}(a)=0$ and $\operatorname{det}((d \mathcal{G} / d \alpha)(a)) \neq 0$, therefore there exists a $T$ periodic solution $\varphi(t, \varepsilon)$ of system $(23)$ satisfying $\varphi(0, \varepsilon) \rightarrow \mathbf{z}_{a}$ as $\varepsilon \rightarrow 0$.

Theorem 5 was proved by Malkin [11] and Roseau [12], for a shorter and new proof see [5].

\section{Proofs of Theorem A and Corollary 1}

The averaging theory we shall use here (see Appendix) deals with smooth system. So, first of all, instead of working with the discontinuous differential system (1) we shall work with the smooth differential system

$$
\begin{aligned}
& x^{\prime}(t)=\omega_{1} y+\varepsilon P_{1 \delta}(t, x, y, z, w)+\varepsilon^{2} R_{1 \delta}(t, x, y, z, w, \varepsilon), \\
& y^{\prime}(t)=-\omega_{1} x+\varepsilon P_{2 \delta}(t, x, y, z, w)+\varepsilon^{2} R_{2 \delta}(t, x, y, z, w, \varepsilon), \\
& z^{\prime}(t)=\omega_{2} w+\varepsilon P_{3 \delta}(t, x, y, z, w)+\varepsilon^{2} R_{3 \delta}(t, x, y, z, w, \varepsilon), \\
& w^{\prime}(t)=-\omega_{2} z+\varepsilon P_{4 \delta}(t, x, y, z, w)+\varepsilon^{2} R_{4 \delta}(t, x, y, z, w, \varepsilon),
\end{aligned}
$$


where

$$
\begin{aligned}
& P_{i \delta}(t, x, y, z, w)=P_{i}^{1}(t, x, y, z, w)+s_{\delta}\left(h_{i}(t, x, y, z, w)\right) P_{i}^{2}(t, x, y, z, w), \\
& R_{i \delta}(t, x, y, z, w, \varepsilon)=F_{i}^{1}(t, x, y, z, w, \varepsilon)+s_{\delta}\left(h_{i}(t, x, y, z, w)\right) F_{i}^{2}(t, x, y, z, w, \varepsilon) .
\end{aligned}
$$

where $s_{\delta}(x)$ is the smooth function defined in Figure 5, such that

$$
\lim _{\delta \rightarrow 0} s_{\delta}(x)=\operatorname{sgn}(x) .
$$
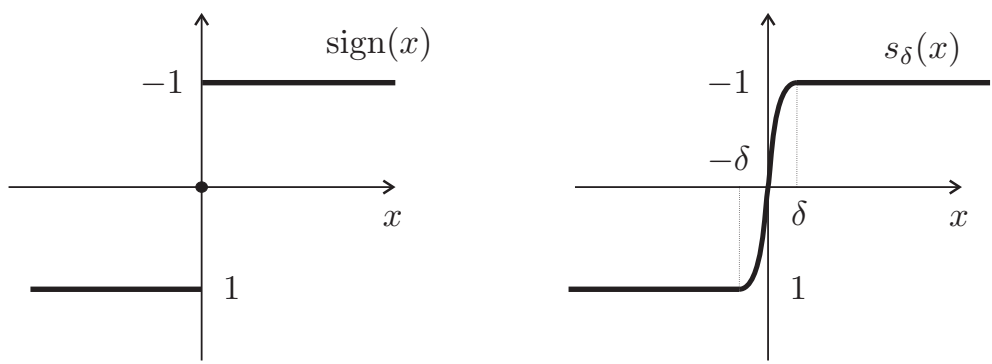

FIGURE 3. The functions $\operatorname{sign}(x)$ and $s_{\delta}(x)$.

Lemma 6. The functions (3) and (6) are periodic solutions of the unperturbed system (27) respectively with periods $T_{1}$ and $T_{2}$.

Proof. Since system (27) with $\varepsilon=0$ is a linear differential system, the proof follows easily.

Proof of Theorem A. It is well known that a Poincaré map defined in a smooth differential system is smooth. So the Poincaré maps associated to the periodic orbits of the differential system (27) are smooth.

The Poincaré maps, restricted at $V$, associated to the periodic solutions of the non-smooth differential system (1), which are perturbations of the periodic solutions (3) are also smooth, indeed, since the orbits starting in $V$ reach the discontinuity set only at points of crossing region (see Appendix), such Poincaré maps are compositions of smooth Poincaré maps. In a similar way it follows that the Poincaré maps, restricted at $V$, associated to the periodic solutions of the non-smooth differential system (1), which are perturbations of the periodic solutions (6) are also smooth.

We can use Theorem 5 (see Section 3) for computing some periodic solutions of the smooth systems. The periodic solutions are zeros of the displacement function, which is the Poincaré map associated to periodic solutions minus the identity. In fact, the non-linear function (26) whose zeros can provide periodic solutions, is the first term of order $\varepsilon$ of the displacement function. See for more details the proof of Theorem 5 in [5].

Since the Poincaré maps associated to periodic solutions of system (1), coming from the perturbed periodic solutions (3) or (6), are smooth and these Poincaré maps are the limit of the Poincaré maps associated to the smooth system (27), for which we can use Theorem 5 , it follows that we also can use Theorem 5 for computing some of the periodic solutions of the non-smooth system (1). In other words, we can apply Theorem 5 to the smooth systems (27) and then pass to the limit, when $\delta \rightarrow 0$, the function (26) for obtaining a function whose zeros can give periodic solutions of the non-smooth system (1). 
We note that system (27) can be written as system (23) taking

$$
\begin{gathered}
\mathbf{x}=\left(\begin{array}{l}
x \\
y \\
z \\
w
\end{array}\right), \quad t=\tau, \quad G_{0}(t, \mathbf{x})=\left(\begin{array}{c}
\omega_{1} y \\
-\omega_{1} x \\
\omega_{2} w \\
-\omega_{2} z
\end{array}\right), G_{1}(t, \mathbf{x})=\left(\begin{array}{c}
P_{1 \delta}(t, \mathbf{x}) \\
P_{2 \delta}(t, \mathbf{x}) \\
P_{3 \delta}(t, \mathbf{x}) \\
P_{4 \delta}(t, \mathbf{x})
\end{array}\right) \\
\text { and } G_{2}(t, \mathbf{x}, \varepsilon)=\left(\begin{array}{c}
R_{1 \delta}(t, \mathbf{x}, \varepsilon) \\
R_{2 \delta}(t, \mathbf{x}, \varepsilon) \\
R_{3 \delta}(t, \mathbf{x}, \varepsilon) \\
R_{4 \delta}(t, \mathbf{x}, \varepsilon)
\end{array}\right) .
\end{gathered}
$$

We shall describe the different elements which appear in the statement of Theorem 5 in the particular case of the differential system (27). Thus we have that $\Lambda=\mathbb{R}^{4}, k=2$ and $n=4$. Let $r_{1}>0$ be arbitrarily small and let $r_{2}>0$ be arbitrarily large. We take the open and bounded subset $V$ of the plane $z=w=0$ as

$$
V=\left\{(x, y, 0,0) \in \mathbb{R}^{4}: r_{1}<\sqrt{x^{2}+y^{2}}<r_{2}\right\}
$$

As usual $\mathrm{Cl}(V)$ denotes the closure of $V$. If $\alpha=(x, y)$, then we can identify $V$ with the set

$$
\left\{\alpha \in \mathbb{R}^{2}: r_{1}<\|\alpha\|<r_{2}\right\}
$$

here $\|\cdot\|$ denotes the Euclidean norm of $\mathbb{R}^{2}$. The function $\beta: \mathrm{Cl}(V) \rightarrow \mathbb{R}^{2}$ is $\beta(\alpha)=(0,0)$. Therefore, in our case the set

$$
\mathcal{Z}=\left\{\mathbf{z}_{\alpha}=(\alpha, \beta(\alpha)), \alpha \in \mathrm{Cl}(V)\right\}=\left\{(x, y, 0,0) \in \mathbb{R}^{4}: r_{1} \leq \sqrt{x^{2}+y^{2}} \leq r_{2}\right\}
$$

Clearly for each $\mathbf{z}_{\alpha} \in \mathcal{Z}$ we can consider the periodic solution $\mathbf{x}\left(t, \mathbf{z}_{\alpha}\right)=\varphi_{1}(t, x, y)$ given by (3) with period $T_{1}$.

Computing the fundamental matrix $M_{\mathbf{z}_{\alpha}}(\tau)$ of the linear differential system (27) with $\varepsilon=0$ associated to the $T$-periodic solution $\mathbf{z}_{\alpha}=(x, y, 0,0)$ such that $M_{\mathbf{z}_{\alpha}}(0)$ be the identity of $\mathbb{R}^{4}$, we get that $M(\tau)=M_{\mathbf{z}_{\alpha}}(\tau)$ is equal to

$$
\left(\begin{array}{cccc}
\cos \left(\omega_{1} t\right) & \sin \left(\omega_{1} t\right) & 0 & 0 \\
-\sin \left(\omega_{1} t\right) & \cos \left(\omega_{1} t\right) & 0 & 0 \\
0 & 0 & \cos \left(\omega_{2} t\right) & \sin \left(\omega_{2} t\right) \\
0 & 0 & -\sin \left(\omega_{2} t\right) & \cos \left(\omega_{2} t\right)
\end{array}\right)
$$


Note that the matrix $M_{\mathbf{z}_{\alpha}}(\tau)$ does not depend on the particular periodic solution $\mathbf{x}\left(\tau, \mathbf{z}_{\alpha}\right)$. Since the matrix

$$
M^{-1}(0)-M^{-1}\left(p T_{1}\right)=\left(\begin{array}{cccc}
0 & 0 & 0 & 0 \\
0 & 0 & 0 & 0 \\
0 & 0 & 2 \sin ^{2}\left(\frac{p \pi \omega_{2}}{\omega_{1}}\right) & \sin \left(\frac{2 p \pi \omega_{2}}{\omega_{1}}\right) \\
0 & 0 & -\sin \left(\frac{2 p \pi \omega_{2}}{\omega_{1}}\right) & 2 \sin ^{2}\left(\frac{p \pi \omega_{2}}{\omega_{1}}\right)
\end{array}\right),
$$

satisfies the assumptions of statement (ii) of Theorem 5. Indeed

$$
\left|\begin{array}{cc}
2 \sin ^{2}\left(\frac{p \pi \omega_{2}}{\omega_{1}}\right) & \sin \left(\frac{2 p \pi \omega_{2}}{\omega_{1}}\right) \\
-\sin \left(\frac{2 p \pi \omega_{2}}{\omega_{1}}\right) & 2 \sin ^{2}\left(\frac{p \pi \omega_{2}}{\omega_{1}}\right)
\end{array}\right|=4 \sin ^{2}\left(\frac{p \pi \omega_{2}}{\omega_{1}}\right) \neq 0 .
$$

because $p \omega_{2} / \omega_{1} \notin \mathbb{Z}$. So we can apply Theorem 5 to system (27).

Now $\xi: \mathbb{R}^{4} \rightarrow \mathbb{R}^{2}$ is $\xi(x, y, z, w)=(x, y)$. We calculate, when $\delta \rightarrow 0$, the function

$$
\mathcal{G}(x, y)=\mathcal{G}(\alpha)=\xi\left(\frac{1}{T_{1}} \int_{0}^{T_{1}} M_{\mathbf{z}_{\alpha}}^{-1}(t) G_{1}\left(t, \mathbf{x}\left(t, \mathbf{z}_{\alpha}\right)\right) d t\right)
$$

and we obtain the function $\mathcal{G}_{1}(x, y)$

(28)

$$
\begin{aligned}
& \frac{1}{T_{1}} \int_{0}^{T_{1}}\left(\cos \left(\omega_{1} t\right) P_{1}^{1}\left(t, \varphi_{1}(t, x, y)\right)-\sin \left(\omega_{1} t\right) P_{2}^{1}\left(t, \varphi_{1}(t, x, y)\right)\right) d t \\
& +\frac{1}{T_{1}} \int_{0}^{T_{1}}\left(\cos \left(\omega_{1} t\right) P_{1}^{2}\left(t, \varphi_{1}(t, x, y)\right)-\sin \left(\omega_{1} t\right) P_{2}^{2}\left(t, \varphi_{1}(t, x, y)\right)\right) \operatorname{sign}\left(h_{1}\left(t, \varphi_{1}(t, x, y)\right)\right) d t
\end{aligned}
$$

and the function $\mathcal{G}_{2}(x, y)$

$$
\begin{aligned}
& \frac{1}{T_{1}} \int_{0}^{T_{1}}\left(\cos \left(\omega_{1} t\right) P_{2}^{1}\left(t, \varphi_{1}(t, x, y)\right)+\sin \left(\omega_{1} t\right) P_{1}^{1}\left(t, \varphi_{1}(t, x, y)\right)\right) d t \\
& +\frac{1}{T_{1}} \int_{0}^{T_{1}}\left(\cos \left(\omega_{1} t\right) P_{2}^{2}\left(t, \varphi_{1}(t, x, y)\right)-\sin \left(\omega_{1} t\right) P_{1}^{2}\left(t, \varphi_{1}(t, x, y)\right)\right) \operatorname{sign}\left(h_{1}\left(t, \varphi_{1}(t, x, y)\right)\right) d t
\end{aligned}
$$

Then, by Theorem 5 we have that for every simple zero $\left(x^{*}, y^{*}\right) \in V$ of the system

$$
\mathcal{G}_{1}(x, y)=0 \quad, \quad \mathcal{G}_{2}(x, y)=0
$$

we have a periodic solution $\varphi(t, \varepsilon)$ of system $(27)$ such that

$$
\varphi(0, \varepsilon) \rightarrow\left(x^{*}, y^{*}, 0,0\right) \quad \text { as } \varepsilon \rightarrow 0 .
$$

Note that system (30) is equivalent to system (9), because both equations only differs in a non-zero multiplicative constant. Hence Theorem A is proved.

Proof of Corollary 1. This proof follows immediately from Theorem A. 


\section{Proofs of Propositions 2 and 3, And Corollary 4}

5.1. Proofs of Propositions. In order to apply Theorem A to system (12) we have to write this system in the standard form (1). For this purpose firstly we follow the steps:

(i) proceed with the change of variable $\phi_{1}=\varepsilon \theta_{1}$ and $\phi_{2}=\varepsilon \theta_{2}$;

$$
\begin{aligned}
\ddot{\theta}_{1}(\tau)= & \frac{1}{\varepsilon} H_{1}\left(\varepsilon \theta_{1}, \varepsilon \dot{\theta}_{1}, \varepsilon \theta_{2}, \varepsilon \dot{\theta}_{2}\right)+F_{1}\left(\tau, \varepsilon \theta_{1}, \varepsilon \dot{\theta}_{1}, \varepsilon \theta_{2}, \varepsilon \dot{\theta}_{2}\right)+\operatorname{sgn}\left(\theta_{1}\right) F_{2}\left(\tau, \varepsilon \theta_{1}, \varepsilon \dot{\theta}_{1}, \varepsilon \theta_{2}, \varepsilon \dot{\theta}_{2}\right) \\
& +\varepsilon\left(G_{1}\left(\tau, \varepsilon \theta_{1}, \varepsilon \dot{\theta}_{1}, \varepsilon \theta_{2}, \varepsilon \dot{\theta}_{2}\right)+\operatorname{sgn}\left(\theta_{1}\right) G_{2}\left(\tau, \varepsilon \theta_{1}, \varepsilon \dot{\theta}_{1}, \varepsilon \theta_{2}, \varepsilon \dot{\theta}_{2}\right)\right)+\mathcal{O}\left(\varepsilon^{3}\right), \\
\ddot{\theta}_{2}(\tau)= & \frac{1}{\varepsilon} H_{2}\left(\varepsilon \theta_{1}, \varepsilon \dot{\theta}_{1}, \varepsilon \theta_{2}, \varepsilon \dot{\theta}_{2}\right)+F_{3}\left(\tau, \varepsilon \theta_{1}, \varepsilon \dot{\theta}_{1}, \varepsilon \theta_{2}, \varepsilon \dot{\theta}_{2}\right)+\operatorname{sgn}\left(\theta_{2}\right) F_{4}\left(\tau, \varepsilon \theta_{1}, \varepsilon \dot{\theta}_{1}, \varepsilon \theta_{2}, \varepsilon \dot{\theta}_{2}\right) \\
& +\varepsilon\left(G_{1}\left(\tau, \varepsilon \theta_{1}, \varepsilon \dot{\theta}_{1}, \varepsilon \theta_{2}, \varepsilon \dot{\theta}_{2}\right)+\operatorname{sgn}\left(\theta_{2}\right) G_{2}\left(\tau, \varepsilon \theta_{1}, \varepsilon \dot{\theta}_{1}, \varepsilon \theta_{2}, \varepsilon \dot{\theta}_{2}\right)\right)+\mathcal{O}\left(\varepsilon^{3}\right) .
\end{aligned}
$$

(ii) expand in Taylor series, for $\varepsilon=0$, the expressions of $\ddot{\theta}_{1}$ and $\ddot{\theta}_{2}$;

(iii) take a new time $t$ given by the rescaling $\tau=\gamma t$, with $\gamma=\sqrt{l_{1} m_{1} /\left(g m_{2}\right)}$;

(iv) and finally, denote

$$
a=\frac{m_{1}+m_{2}}{m_{2}}>1 \quad \text { and } \quad b=\frac{l_{1}\left(m_{1}+m_{2}\right)}{l_{2} m_{2}}>0 .
$$

Hence, we obtain the following equations of motion for the double pendulum

$$
\begin{aligned}
\theta_{1}^{\prime \prime}(t)= & -a \theta_{1}+\theta_{2}+\varepsilon\left(\tilde{F}_{1}\left(t, \theta_{1}, \theta_{1}^{\prime}, \theta_{2}, \theta_{2}^{\prime}\right)+\tilde{G}_{1}(t)\right) \\
& +\varepsilon \operatorname{sgn}\left(\theta_{1}\right)\left(\tilde{F}_{2}\left(t, \theta_{1}, \theta_{1}^{\prime}, \theta_{2}, \theta_{2}^{\prime}\right)+\tilde{G}_{2}(t)\right)+\mathcal{O}\left(\varepsilon^{2}\right), \\
\theta_{2}^{\prime \prime}(t)= & b \theta_{1}-b \theta_{2}+\varepsilon\left(\tilde{F}_{3}\left(t, \theta_{1}, \theta_{1}^{\prime}, \theta_{2}, \theta_{2}^{\prime}\right)+\tilde{G}_{3}(t)\right) \\
& +\varepsilon \operatorname{sgn}\left(\theta_{2}\right)\left(\tilde{F}_{4}\left(t, \theta_{1}, \theta_{1}^{\prime}, \theta_{2}, \theta_{2}^{\prime}\right)+\tilde{G}_{4}(t)\right)+\mathcal{O}\left(\varepsilon^{2}\right),
\end{aligned}
$$

where now the prime denotes derivative with respect to the new time $t$. Here the functions $\tilde{F}_{i}$, and $\tilde{G}_{i}$ for $i=1,2,3,4$ are given in (16) and (17). It is worth to say that the parameters $\gamma, a$ and $b$ defined above are the same defined in (13).

Introducing the variables $(X, Y, Z, W)=\left(\theta_{1}, \theta_{1}^{\prime}, \theta_{2}, \theta_{2}^{\prime}\right)$ we write the differential system of the non-smooth perturbed double pendulum (31) as a first-order differential system defined in $\mathbb{R}^{4}$. Thus we have the differential system

$$
\begin{aligned}
X^{\prime}(t)= & Y, \\
Y^{\prime}(t)= & -a X+Z+\varepsilon\left(\tilde{F}_{1}(t, X, Y, Z, W)+\tilde{G}_{1}(t)\right) \\
& +\varepsilon \operatorname{sign}(X)\left(\tilde{F}_{2}(t, X, Y, Z, W)+\tilde{G}_{2}(t)\right)+\mathcal{O}\left(\varepsilon^{2}\right), \\
Z^{\prime}(t)=\quad & W, \\
W^{\prime}(t)= & b X-b Z+\varepsilon\left(\tilde{F}_{3}(t, X, Y, Z, W)+\tilde{G}_{3}(t)\right) \\
& +\varepsilon \operatorname{sign}(Z)\left(\tilde{F}_{4}(t, X, Y, Z, W)+\tilde{G}_{4}(t)\right)+\mathcal{O}\left(\varepsilon^{2}\right) .
\end{aligned}
$$

Finally, we write system (32) in such a way that the linear part at the origin of the unperturbed system will be in its real normal Jordan form. Then, doing the change of variables 
$(t, X, Y, Z, W) \rightarrow(t, x, y, z, w)$ given by

$$
\left(\begin{array}{l}
x \\
y \\
z \\
w
\end{array}\right)=\left(\begin{array}{cccc}
\frac{b \omega_{1}}{\sqrt{\Delta}} & 0 & \frac{\omega_{1}(a-b+\sqrt{\Delta})}{2 \sqrt{\Delta}} & 0 \\
0 & \frac{b}{\sqrt{\Delta}} & 0 & \frac{a-b+\sqrt{\Delta}}{2 \sqrt{\Delta}} \\
-\frac{b \omega_{2}}{\sqrt{\Delta}} & 0 & \frac{\omega_{2}(-a+b+\sqrt{\Delta})}{2 \sqrt{\Delta}} & 0 \\
0 & -\frac{b}{\sqrt{\Delta}} & 0 & \frac{-a+b+\sqrt{\Delta}}{2 \sqrt{\Delta}}
\end{array}\right)\left(\begin{array}{c}
X \\
Y \\
Z \\
W
\end{array}\right)
$$

the differential system (32) becomes

$$
\begin{aligned}
x^{\prime}= & \omega_{1} y, \\
y^{\prime}= & -\omega_{1} x+\varepsilon \frac{1}{2 \sqrt{\Delta}}\left(2 b\left(\check{F}_{1}+\tilde{G}_{1}(t)+\left(\check{F}_{2}+\tilde{G}_{2}(t)\right) \operatorname{sign}(\mathcal{A})\right)\right) \\
& +\varepsilon \frac{1}{2 \sqrt{\Delta}}\left((a-b+\sqrt{\Delta})\left(\check{F}_{3}+\tilde{G}_{3}(t)+\left(\check{F}_{4}+\tilde{G}_{4}(t)\right) \operatorname{sign}(\mathcal{C})\right)\right)+\mathcal{O}\left(\varepsilon^{2}\right), \\
z^{\prime}= & \omega_{2} w, \\
w^{\prime}= & -\omega_{2} z+\varepsilon \frac{1}{2 \sqrt{\Delta}}\left(-2 b\left(\check{F}_{1}+\tilde{G}_{1}(t)+\left(\check{F}_{2}+\tilde{G}_{2}(t)\right) \operatorname{sign}(\mathcal{A})\right)\right) \\
& +\varepsilon \frac{1}{2 \sqrt{\Delta}}\left((-a+b+\sqrt{\Delta})\left(\check{F}_{3}+\tilde{G}_{3}(t)+\left(\check{F}_{4}+\tilde{G}_{4}(t)\right) \operatorname{sign}(\mathcal{C})\right)\right)+\mathcal{O}\left(\varepsilon^{2}\right),
\end{aligned}
$$

where $\check{F}_{i}(t, x, y, z, w)=\tilde{F}_{i}(t, \mathcal{A}, \mathcal{B}, \mathcal{C}, \mathcal{D})$ for $i=1,2,3,4$ with

$$
\begin{aligned}
& \mathcal{A}=\frac{(-a+b+\sqrt{\Delta})}{2 b \omega_{1}} x-\frac{(a-b+\sqrt{\Delta})}{2 b \omega_{2}} z, \\
& \mathcal{B}=\frac{(-a+b+\sqrt{\Delta})}{2 b} y-\frac{(a-b+\sqrt{\Delta})}{2 b} w, \\
& \mathcal{C}=\frac{1}{\omega_{1}} x+\frac{1}{\omega_{2}} z, \\
& \mathcal{D}=y+w .
\end{aligned}
$$

Proof of Proposition 2. Computing the functions (4) and (5) for the differential system (34) we obtain the functions given in (18). Consequently, the system of functions (9) is equivalent to the system of functions (21). Then, by Theorem A we have that for every simple zero $\left(x^{*}, y^{*}\right)$ of the system $(21)$ there exists a periodic solution $(x, y, z, w)(t, \varepsilon)$ of $(34)$ such that

$$
(x, y, z, w)(0, \varepsilon) \rightarrow\left(x^{*}, y^{*}, 0,0\right) \quad \text { as } \varepsilon \rightarrow 0 .
$$


Going back through the change of coordinates (33) we get a periodic solution $(X, Y, Z, W)(t, \varepsilon)$ of system (32) such that

$$
\left(\begin{array}{c}
X(t, \varepsilon) \\
Y(t, \varepsilon) \\
Z(t, \varepsilon) \\
W(t, \varepsilon)
\end{array}\right) \rightarrow\left(\begin{array}{c}
\frac{-a+b+\sqrt{\Delta}}{2 b \omega_{1}}\left(x^{*} \cos \left(\omega_{1} t\right)+y^{*} \sin \left(\omega_{1} t\right)\right) \\
\frac{-a+b+\sqrt{\Delta}}{2 b}\left(y^{*} \cos \left(\omega_{1} t\right)-x^{*} \sin \left(\omega_{1} t\right)\right) \\
\frac{1}{\omega_{1}}\left(x^{*} \cos \left(\omega_{1} t\right)+y^{*} \sin \left(\omega_{1} t\right)\right) \\
y^{*} \cos \left(\omega_{1} t\right)-x^{*} \sin \left(\omega_{1} t\right)
\end{array}\right)
$$

as $\varepsilon \rightarrow 0$. Consequently we obtain a periodic solution $\varphi(\tau, \varepsilon)$ of system (12) such that

$$
\varphi(\tau, \varepsilon)=\left(\varepsilon X\left(\frac{\tau}{\gamma}, \varepsilon\right), \frac{\varepsilon}{\gamma} Y\left(\frac{\tau}{\gamma}, \varepsilon\right), \varepsilon Z\left(\frac{\tau}{\gamma}, \varepsilon\right), \frac{\varepsilon}{\gamma} W\left(\frac{\tau}{\gamma}, \varepsilon\right)\right),
$$

which is clearly $\gamma T_{1}$-periodic. Moreover $\varphi(0, \varepsilon) \rightarrow(0,0,0,0)$ when $\varepsilon \rightarrow 0$. Hence Theorem $\mathrm{A}$ is proved.

Proof of Proposition 3. Computing the functions (7) and (8) to the differential system (34) we obtain the functions given in (20). Consequently, the system of functions (10) is equivalent to the system of functions (22). Then, by Corollary 1 we have that for every simple zero $\left(z^{*}, w^{*}\right)$ of the system of functions $(22)$ there exists a periodic solution $(x, y, z, w)(t, \varepsilon)$ of $(34)$ such that

$$
(x, y, z, w)(0, \varepsilon) \rightarrow\left(0,0, z^{*}, w^{*}\right) \quad \text { as } \varepsilon \rightarrow 0 .
$$

From here, the proof follows analogously to the proof of Proposition 2.

5.2. Proof of Corollary. To obtain the expression of the functions given in (18) and (20) we have to study the changes of sign of the functions $\pi_{1} \circ \varphi_{1}(t, x, y)$ (defined in (3)) and $\pi_{3} \circ \varphi_{2}(t, z, w)$ (defined in (6)) respectively for $t \in\left[0, T_{1}\right]$ and $t \in\left[0, T_{2}\right]$.

Note that $\pi_{1} \circ \varphi_{1}(t, x, y)=0$ only for

$$
t_{n}=\frac{1}{\omega_{1}}\left(\pi n-\arctan \left(\frac{x}{y}\right)\right) .
$$

So, if $x y<0$, then $t_{n} \in\left[0, T_{1}\right]$ only for $n=0,1$; and if $x y>0$, then $t_{n} \in\left[0, T_{1}\right]$ only for $n=1,2$. We know that for all $t \in\left[t_{n}, t_{n+1}\right]$ the function $\pi_{1} \circ \varphi_{1}(t, x, y)$ has the same sign and different sign of any $t \in\left[t_{n-1}, t_{n}\right]$, thus the integral can be computed using the partitions $\left\{0, t_{0}, t_{1}, T_{1}\right\}$ when $x y<0$ and $\left\{0, t_{1}, t_{2}, T_{1}\right\}$ when $x y>0$ as the limits of integration.

The study of changes of the sign of the function $\pi_{3} \circ \varphi_{2}(t, z, w)$ for $t \in\left[0, T_{2}\right]$ and $z w \neq 0$ is completely analogous.

Proof of Corollary 4. Firstly, we have to check the crossing hypothesis for the system (12) or equivalently for the system (32). Note that we have four different vector fields defined in four different regions (see Figure 4).

In the region $\mathcal{R}_{1}=\{X>0$ and $Z>0\}$ we have

$$
\mathcal{X}_{1}=\left(\begin{array}{l}
Y \\
-a X+Z+\varepsilon\left(1+k_{1} Z\right) \\
W \\
b X-b Z+\varepsilon\left(1+k_{3} Z\right),
\end{array}\right) .
$$


In the region $\mathcal{R}_{2}=\{X<0$ and $Z>0\}$ we have

$$
\mathcal{X}_{2}=\left(\begin{array}{l}
Y \\
-a X+Z-\varepsilon\left(1-k_{1} Z\right) \\
W \\
b X-b Z+\varepsilon\left(1+k_{3} Z\right),
\end{array}\right) .
$$

In the region $\mathcal{R}_{3}=\{X<0$ and $Z<0\}$ we have

$$
\mathcal{X}_{3}=\left(\begin{array}{l}
Y \\
-a X+Z-\varepsilon\left(1-k_{1} Z\right) \\
W \\
b X-b Z-\varepsilon\left(1-k_{3} Z\right),
\end{array}\right) .
$$

Finally, in the region $\mathcal{R}_{4}=\{X>0$ and $Z<0\}$ we have

$$
\mathcal{X}_{4}=\left(\begin{array}{l}
Y \\
-a X+Z+\varepsilon\left(1+k_{1} Z\right) \\
W \\
b X-b Z-\varepsilon\left(1-k_{3} Z\right),
\end{array}\right) .
$$

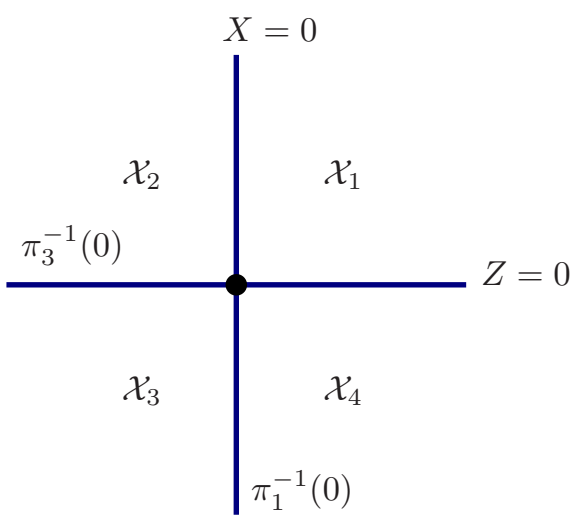

FiguRE 4. Four different vector fields.

To study the types of the sets $\mathcal{M}_{i j}$ (see Appendix), we have to compute Lie derivative of the functions $\pi_{1}$ and $\pi_{3}$ with respect to the vector fields $\mathcal{X}_{i}$ for $i=1,2,3$, 4 , i.e.

$$
\left(\mathcal{L}_{\mathcal{X}_{i}}\right)\left(\pi_{j}\right)(X, Y, Z, W)=\left\langle\nabla \pi_{j}, \mathcal{X}_{i}\right\rangle(X, Y, Z, W),
$$

where $\pi_{j}$ is the projection onto the $j$ th coordinate. 
Proceeding with these calculations we have

$$
\begin{aligned}
& \left(\mathcal{L}_{\mathcal{X}_{1}}\right)\left(\pi_{1}\right)(X, Y, Z, W)=\left(\mathcal{L}_{\mathcal{X}_{2}}\right)\left(\pi_{1}\right)(X, Y, Z, W)=Y, \\
& \left(\mathcal{L}_{\mathcal{X}_{2}}\right)\left(\pi_{2}\right)(X, Y, Z, W)=\left(\mathcal{L}_{\mathcal{X}_{3}}\right)\left(\pi_{2}\right)(X, Y, Z, W)=W \\
& \left(\mathcal{L}_{\mathcal{X}_{3}}\right)\left(\pi_{1}\right)(X, Y, Z, W)=\left(\mathcal{L}_{\mathcal{X}_{4}}\right)\left(\pi_{1}\right)(X, Y, Z, W)=Y, \\
& \left(\mathcal{L}_{\mathcal{X}_{1}}\right)\left(\pi_{2}\right)(X, Y, Z, W)=\left(\mathcal{L}_{\mathcal{X}_{4}}\right)\left(\pi_{2}\right)(X, Y, Z, W)=W .
\end{aligned}
$$

Hence we can conclude that in the set

$$
\mathcal{T}=\{(X, Y, 0,0)\} \bigcup\{(0,0, Z, W)\},
$$

the flow is tangent to the discontinuous set, and in any other point the flow cross the set of discontinuity.

In the coordinates defined in (33), we have that

$$
\mathcal{T}=\left\{\left(x, y,-\frac{\omega_{2}}{\omega_{1}} x,-y\right)\right\} \bigcup\left\{\left(x, y, \beta \frac{\omega_{2}}{\omega_{1}} x, \beta y\right)\right\},
$$

where

$$
\beta=-\frac{a-b-\sqrt{\Delta}}{a-b+\sqrt{\Delta}} .
$$

Observe that the periodic orbits given by Lemma 6 filling the planes $\{(x, y, 0,0)\}$ and $\{(0,0, z, w)\}$, except the origin, do not reach the set $\mathcal{T}$. Thus, for $|\varepsilon|>0$ sufficiently small, there exists a neighborhood of $\{(x, y, 0,0)\} \backslash(0,0,0,0)$ and $\{(0,0, z, w)\} \backslash(0,0,0,0)$ such that the orbits cross the set of discontinuity. In other words, the crossing hypothesis is satisfied for every $\varepsilon \in\left(-\varepsilon_{0}, \varepsilon_{0}\right)$ for some $\varepsilon_{0}>0$.

Now assume that the function are $\gamma T_{1}$-periodic in the variable $\tau$. By studying the changes of the sign of the function $\pi_{1} \circ \varphi_{1}(t, x, y)$ for $t \in\left[0, T_{1}\right]$ we conclude that the functions (18) and (20) are given by

$$
\begin{aligned}
& \mathcal{F}_{1}(x, y)=\left\{\begin{array}{cc}
y+\frac{4(a+b+\sqrt{\Delta})}{\omega_{1} \sqrt{1+\frac{x^{2}}{y^{2}}}} & \text { if } \quad y>0, \\
y-\frac{4(a+b+\sqrt{\Delta})}{\omega_{1} \sqrt{1+\frac{x^{2}}{y^{2}}}} & \text { if } \quad y<0,
\end{array}\right. \\
& \mathcal{F}_{2}(x, y)=\left\{\begin{array}{c}
x+\frac{4 x(a+b+\sqrt{\Delta})}{\omega_{1} y \sqrt{1+\frac{x^{2}}{y^{2}}}} \quad \text { if } \quad y>0, \\
x-\frac{4 x(a+b+\sqrt{\Delta})}{\omega_{1} y \sqrt{1+\frac{x^{2}}{y^{2}}}} \quad \text { if } y<0,
\end{array}\right.
\end{aligned}
$$

So the system $\mathcal{F}_{1}(x, y)=0$ and $\mathcal{F}_{2}(x, y)=0$ has the following simple solutions:

$$
\left(x_{1}^{*}, y_{1}^{*}\right)=\left(0,4 \frac{a+b+\sqrt{\Delta}}{\omega_{1}}\right),
$$


and

$$
\left(x_{2}^{*}, y_{2}^{*}\right)=\left(0,-4 \frac{a+b+\sqrt{\Delta}}{\omega_{1}}\right) .
$$

Hence, by Theorem A we have two $\gamma T_{1}$-periodic solution of the non-smooth perturbed double pendulum. The argument in the case when the functions are $\gamma T_{2}$-periodic is completely analogous. So we have conclude the proof of corollary.

\section{Appendix: Basic COnCEPts on Filippov systems}

We say that a vector field $X: D \subset \mathbb{R}^{n} \rightarrow \mathbb{R}^{n}$ is piecewise continuous if its domain of definition $D$ can be partitioned in a finite collection of connected, open and disjoint sets $D_{i}$, $i=1, \cdots, k$, such that $\cup \overline{D_{i}}=D$, and the vector field $\left.X\right|_{\bar{D}_{i}}$ is continuous for $i=1, \cdots, k$.

We denote by $S_{X} \subset \partial D_{1} \cup \cdots \cup \partial D_{k}$ the set of points where the vector field $X$ is discontinuous. By assumptions, the set $S_{X}$ has measure zero.

If $\Sigma \subset S_{X}$ is a manifold of codimension one, then $\Sigma$ can be decomposed as the union of the closure of the following three kind of regions (see Figure 5):

$$
\begin{aligned}
& \Sigma^{c}=\{x \in \Sigma:(X h)(Y h)(x)>0\} ; \\
& \Sigma^{e}=\{x \in \Sigma:(X h)(x)>0 \text { e }(Y h)(x)<0\} ; \\
& \Sigma^{s}=\{x \in \Sigma:(X h)(x)<0 \text { e }(Y h)(x)>0\} .
\end{aligned}
$$
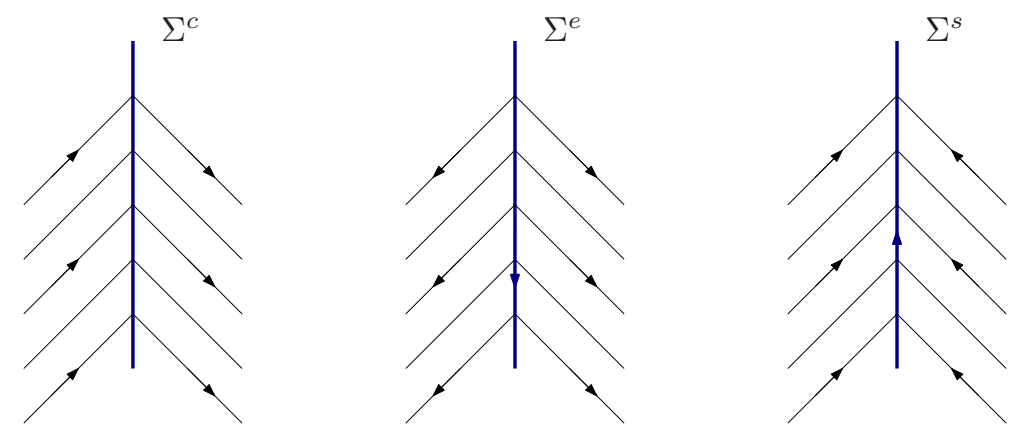

FiguRE 5. Crossing region $\left(\Sigma^{c}\right)$, escaping region $\left(\Sigma^{e}\right)$ and sliding region $\left(\Sigma^{s}\right)$.

For $p \in \Sigma^{e} \cup \Sigma^{s}$ we define the Sliding Vector Field as

$$
Z_{s}(p)=\frac{1}{(Y h)(p)-(X h)(p)}((Y h)(X h)(p)-(X h)(Y h)(p)) .
$$

Consider the following equation

$$
\dot{x}=X(x),
$$

where $X: D \subset \mathbb{R}^{n} \rightarrow \mathbb{R}^{n}$ is a piecewise continuous vector field. The local solution of the equation (36) passing through a point $p \in \Sigma$ is given by the Filippov convention:

(i) for $p \in \Sigma^{c}$ such that $(X h)(p),(Y h)(p)>0$ and taking the origin of time at $p$, the trajectory is defined as $\varphi_{Z}(t, p)=\varphi_{Y}(t, p)$ for $t \in I_{p} \cap\{t<0\}$ and $\varphi_{Z}(t, p)=\varphi_{X}(t, p)$ for $t \in I_{p} \cap\{t>0\}$. For the case $(X h)(p),(Y h)(p)<0$ the definition is the same reversing time; 
(i) for $p \in \Sigma^{e} \cup \Sigma^{s}$ such that $Z_{s}(p) \neq 0, \varphi_{Z}(t, p)=\varphi_{Z_{s}}(t, p)$ for $t \in I_{p} \subset \mathbb{R}$.

Here $\varphi_{W}$ denotes the flow of a vector field $W$.

For more details about discontinuous differential equation see Filippov's book [7].

\section{ACKNOWLEDGEMENTS}

The first author is partially supported by a MINECO/FEDER grant MTM2008-03437, an AGAUR grant number 2009SGR-0410, an ICREA Academia and FP7-PEOPLE-2012IRSES-316338 and 318999. The second author is partially supported by a FAPESP-BRAZIL grant 2012/10231-7. The third author is partially supported by a FAPESP-BRAZIL grant 2007/06896-5. The first and third authors are also supported by the joint project CAPESMECD grant PHB-2009-0025-PC.

\section{REFERENCES}

[1] A. A. Andronov, A.A. Vitt E S. E. Khaikin, Theory of oscillators, International Series of Monographs In Physics 4, Pergamon Press, 1966.

[2] M. Di Bernardo et AL., Bifurcation in nonsmooth dynamical systems, Publications of the Bristol Centre for Applied Nonlinear Mathematics 4, (2005), 629-701.

[3] N. N. Bogoliubov, On some statistical methods in mathematical physics, Izv. vo Akad. Nauk Ukr. SSR, Kiev, 1945.

[4] N. N. Bogoliubov and N. Krylov, The application of methods of nonlinear mechanics in the theory of stationary oscillations, Publ. 8 of the Ukrainian Acad. Sci. Kiev, 1934.

[5] A. Buică, J.P. Françoise and J. Llibre, Periodic solutions of nonlinear periodic differential systems with a small parameter, Communication on Pure and Applied Analysis 6 (2007), 103-111.

[6] P. Fatou, Sur le mouvement d'un systàme soumis à des forces à courte période, Bull. Soc. Math. France 56 (1928), 98-139

[7] A. F. Filippov, Differential equations with discontinuous righthand side, Mathematics and Its Applications, Kluwer Academic Publishers, Dordrecht, 1988.

[8] H. Iro, A modern approach to classical mechanics, World Scientific Publishing Co., Inc., River Edge, NJ, 2002.

[9] J. Llibre, D.D. Novaes And M.A. Teixeira, On the periodic solutions of a perturbed double pendulum, São Paulo J. Math Sciences IME-USP 5 (2011), 317-330

[10] J. Llibre And M.A. Teixeira, On the stable limit cycle of a weight-driven pendulum clock, Eur. J. Phys. 31 (2010), 1249-1254

[11] I.G. Malkin, Some problems of the theory of nonlinear oscillations, (Russian) Gosudarstv. Izdat. Tehn.Teor. Lit., Moscow, 1956.

[12] M. Roseau, Vibrations non linéaires et théorie de la stabilité, (French) Springer Tracts in Natural Philosophy, Vol.8 Springer-Verlag, Berlin-New York, 1966.

[13] J. A. Sanders F. Verhulst and J. Murdock, Averaging Methods in Nonlinear Dynamical Systems, Second edition, Applied Mathematical Sciences 59, Springer, New York, 2007.

[14] J. Sotomayor and M.A. Teixeira, Regularization of Discontinuous Vector Field, International Conference on Differential Equation, Lisboa, 1995, World Sci. Publ., River Edge, NJ, (1998), pp 207223.

1 Departament de Matematiques, Universitat Autònoma de Barcelona, 08193 Bellaterra, Barcelona, Catalonia, Spain

E-mail address: jllibre@mat.uab.cat

2 Departamento de Matematica, Universidade Estadual de Campinas, Caixa Postal 6065, 13083 859, CAmpinas, SP, Brazil

E-mail address: ddnovaes@gmail.com, teixeira@ime.unicamp.br 